ribosome during initiation and then is free to bind to and dissociate a $70 \mathrm{~S}$ ribosome leaving a messenger, or the $\mathrm{F}_{3}$ molecules may remain bound, one per $30 \mathrm{~S}$ subunit, throughout the ribosome cycle. If the latter is the case, ribosomes should dissociate at the termination of a round of protein synthesis; furthermore, the dissociating activity of the factor must be blocked by an aminoacyl or peptidyl-tRNA, otherwise $70 \mathrm{~S}$ ribosomes would never form. Deciding between these alternatives (and the odds are on the former) will complete the picture of the ribosome cycle in bacterial cells.

\section{REVERSE TRANSCRIPTASES}

\section{Roundabouts and Swings}

from our Cell Biology Correspondent

Ox Monday, December 14, Dr Sol Spiegelman, lccturing at the Royal College of Surgeons at the invitation of the Imperial Cancer Research Fund, treated his audience to an adroit review of the prodigious amount of research which he and his colleagues have done on reverse transcriptases in animal cancer viruses and human cancer cells. After briefly discussing the three possible models which account for the replication of RNA viruses in a cellular environment dominated by DNA, and outlining his work on the replication of the phages, Dr Spiegelman reached the meat of his lecture - the significance of the discovery of reverse transcriptases to cancer research and the search for a human RNA leukaemia virus.

The presence of reverse transcriptases in animal tumour viruses makes possible the synthesis of a highly radioactive labelled DNA with a base sequence complementary to that of a tumour virus RNA. If human tumour viruses exist, and if they are related in their base sequences to the animal tumour viruses, such DNA might be an effective probe, in hybridization experiments, for a human tumour virus nucleic acid in a cancer cell. But unfortunately, Spiegelman and his colleagues found in hybridization tests that the base sequences of the various animal tumour viruses are not closely related. This slightly disappointing result means, of course, that there is no good reason to anticipate a close relationship between the base sequences of a putative human tumour virus and any of the animal tumour viruses. DNA complementary to tumour virus RNA is therefore a much blunter probe than cverybody hoped it might prove to be.

Synthetic double stranded RNAs and DNAs and RNA/DNA hybrid molecules, on the other hand, have been shown to be more useful than anybody had a right to expect. Certain synthetic nucleic acids are by about two orders of magnitude better templates than any natural nucleic acids so far tested for reverse transcriptases in animal tumour viruses. Armed with this information, Spiegelman et al. began to search for polymerase activities, first in the plasma of human leukaemia patients and then in extracts of leukaemia cells. In the cells of more than forty leukaemics, they have now found a polymerase activity which uses poly $\mathrm{dT} \cdot$ poly rA as a template, as well as an activity which replicates poly $\mathrm{dC}$.poly $\mathrm{dG}$. This latter activity seems to be present in all proliferating cells irrespective of whether or not they are malignant, but the former activity, which utilizes an RNA/DNA hybrid as a template, seems to be restricted to cancer cells. In addition to being present in leukaemia cells, it has been detected in the cells of two osteosarcomas and one chondrosarcoma. It is interesting, however, that only one of seven Burkitt's lymphoma biopsies, supplied by Dr G. Klein, contained detectable amounts of the poly dT.poly rA polymerase, whereas all contained the activity which replicates poly $\mathrm{dC} \cdot$ poly $\mathrm{d} G$.

So far, as Dr Spiegelman took pains to stress, neither his nor anybody else's work on the nucleic acid polymerases in human cancer cells proves the existence of human leukaemia or sarcoma viruses. The polymerase activity which distinguishes human cancer cells from other non-malignant, but proliferating cells, differs markedly from the reverse transcriptase in animal tumour viruses; the two activities can, for example, be differentiated on the basis of their preferences for synthetic templates of various base compositions. But irrespective of any question of the viral aetiology of human cancers, the presence in cancer cells of a polymerase activity which can use a hybrid nucleic acid as a template provides a new tool for diagnosis of cancer and for following the course of remissions and relapses during therapy.

\section{PROTEIN SYNTHESIS}

\section{Inside and Outside the Ribosome}

\section{from our Nucleic Acids Correspondent}

STUDIES of the structure and assembly of the ribosomal particles from Escherichia coli continue to burgeon, and a rich crop is to be found in the November issue of the Proceedings of the National Academy of Sciences. Among these articles are the striking results of Chang and Flaks (Proc. US Nat. Acad. Sci., 67, 1321; 1970) and Craven and Gupta $\left(\mathbf{6}^{\mathbf{4}}, \mathbf{1 3 2 9} ; \mathbf{1 9 7 0}\right)$, who have attempted to examine the topography of $30 \mathrm{~S}$ ribosomal particles by partially digesting them with low concentrations of trypsin or treating them with protein reagents. Chang and Flaks reasoned that if some of the ribosomal proteins are more accessible to the enzyme than others, these will be most rapidly digested. Hence it might be possible to deduce the relative accessibility of the proteins (those which are external and those which are not) in the particle. They therefore analysed the proteins remaining in variously digested ribosomal particles by polyacrylamide gel electrophoresis and compared them with the proteins from undigested controls run in parallel. They report the remarkable finding that those proteins which become associated early with the RNA in the course of the reconstitution scheme for the 30 S particles described by Nomura are usually the least sensitive to digestion. Those proteins added at intermediate or late times in the reconstitution are, in contrast, digested more easily. In other words, there is an inverse relationship between the sensibility to digestion of the ribosomal proteins and the order in which they map in the reconstitution of the $30 \mathrm{~S}$ ribosomal particles. Although thcre were a few discrepancies and ambiguities, there is, nevertheless, a close agreement to this model considering the relatively unsophisticated method used. 